\title{
The Steady-State System Size Distribution for a Modified D-Policy Geo/G/1 Queueing System
}

\author{
Renbin Liu and Zhaohui Deng \\ School of Mathematics and Statistics, Chongqing University of Technology, Chongqing 400054, China \\ Correspondence should be addressed to Renbin Liu; liurb888@126.com
}

Received 26 March 2014; Revised 3 June 2014; Accepted 3 June 2014; Published 22 June 2014

Academic Editor: Alexander N. Dudin

Copyright (C) 2014 R. Liu and Z. Deng. This is an open access article distributed under the Creative Commons Attribution License, which permits unrestricted use, distribution, and reproduction in any medium, provided the original work is properly cited.

\begin{abstract}
This paper examines a discrete-time modified D-policy Geo/G/1 queue with Bernoulli feedback. Using a decomposition method, the steady-state system size distribution at epoch $n^{+}$is obtained. Moreover, the steady-state system size distributions at epochs $n^{-}$ and $n$ are also derived. Two special cases are given. Finally, a wireless local area network is numerically presented to validate the applicability of steady-state system size distribution and its important application in system capacity design.
\end{abstract}

\section{Introduction}

The D-policy of queueing systems has been extensively studied in lots of research papers for the past decades. In contrast with the N-policy of Yadin and Naor [1] and the T-policy of Heyman [2], the D-policy controls the queueing system by the workload of waiting customers (i.e., the server resumes its service only when the sum of the service times of all waiting customers exceeds a positive constant $D$ ). The Dpolicy can be used in many real-world queueing systems to control the number of server startup and thereby reduces the average cost of system.

The D-policy M/G/1 queue was first proposed by Balachandran [3] and Balachandran and Tijms [4]. Later on, this kind of queue was discussed by Boxma [5], Tijms [6], Artalejo [7], Gakis et al. [8], and Rhee [9]. The other works on the continuous-time D-policy queues can be found in [10-15] and references therein. For related literature on discrete-time $D$-policy queues the reader may refer to [16]. Recently, Lee et al. [17] analyzed the discrete-time MAP/G/1 queue under the $\mathrm{D}$-policy and derived the probability generating functions of the queue length, workload, waiting time, and sojourn time distributions. Wei et al. [18] dealt with the transient and equilibrium properties of the queue length for a discrete-time Geo/G/1 queueing system with delayed D-policy and gave the stochastic decomposition of the queue length in equilibrium. However, compared with the continuous-time counterparts, the discrete-time D-policy queueing systems received less attention in the literature.

The study of feedback queues was initiated by Takács [19]. The papers of Choi et al. [20], Choi and Kim [21], and Kumar and Raja [22] and their references provided us a wide study of different continuous-time feedback queues. Atencia and Moreno [23] first introduced a discrete-time queue with feedback. The phenomenon of feedback in the queueing systems occurs in many practical situations. For instance, in telecommunication systems, when messages are received with errors at the destination, they are resent. In a call center, users may repeat calls when they do not completely solve their problems and so forth. To the best of our knowledge, the existing literature on feedback queues focuses mainly on the continuous case. Little work has appeared in the discretetime research area.

In spite of the importance of D-policy and feedback in queueing models, there is only very little literature concerned with D-policy queues with feedback. Moreover, as far as we know, there is no work about the discrete-time D-policy queues with feedback. On the other hand, the existing Dpolicy queues focus on the probability generating function (PGF) of steady-state system size rather than the steady-state system size distribution. The first objective in this paper is to introduce a discrete-time modified D-policy Geo/G/1 queueing system with Bernoulli feedback, in which $D$ is a fixed positive integer value. Next, in such a queue, we will obtain 
the steady-state system size distributions at epochs $n^{+}, n^{-}$, and $n$, where $n^{-}$and $n^{+}$represent $\lim _{\Delta t \rightarrow 0}(n-|\Delta t|)$ and $\lim _{\Delta t \rightarrow 0}(n+|\Delta t|)$, respectively. Lastly, we numerically present the applicability and application of steady-state system size distributions.

Our queueing system can be used to model many practical situations. For example, in a wireless local area network (WLAN), the access point (AP) is a specially configured node on WLAN and acts as a central receiver and transmitter of WLAN radio signals. Once the radio signals are sent to the AP, they will be identified and transmitted if the AP is free. When the AP is busy, the received radio signals are placed in queue. Because of power saving, the AP is designed to start serving exhaustively whenever the workload of radio signals reaches a predetermined level. When a radio signal is transmitted with errors at the destination, it is retransmitted. In view of the fact that the receptions and transmissions of radio signals are proceeded in fixed time intervals, this kind of WLAN can be modeled as a discrete-time modified D-policy queue with feedbacks. Moreover, for the above WLAN, the steady-state system size distribution obtained in Section 3 is very useful in network capacity design (see Section 5).

This paper is organized as follows. The next section presents assumptions and preliminaries. Section 3 analyzes the PGF of transient-state system size distribution. Based on this PGF the steady-state system size distributions at epochs $n^{+}, n^{-}$, and $n$ are obtained. Sections 4 and 5 give two special cases and numerically illustrate the applicability and important application of steady-state system size distribution for a WLAN. Conclusions are drawn in Section 6.

\section{Queue Assumptions and Some Preliminaries}

We consider a discrete-time modified D-policy Geo/G/1 queueing system with Bernoulli feedback where the time is divided into constant length intervals (called slots). It is well known that the probability of an arrival and a departure occurring simultaneously is not zero in discrete time. This probability is positive in the discrete-time setting. That is why the order of the arrivals and departures must be stated. Usually, there are two models in discrete-time queues, that is, late arrival system with delayed access (LAS-DA) and early arrival system (EAS), which are also known as arrival first (AF) and departure first (DF) policies, respectively. These concepts and detailed discussions can be found in Hunter [24]. Here, we only discuss the mentioned model with LAS-DA; that is, a potential arrival can only take place in $\left(n^{-}, n\right), n=0,1,2, \ldots$, and a potential departure or feedback can only take place in $\left(n, n^{+}\right), n=1,2, \ldots$. We do not permit a departure at such time point for a customer who has just arrived the instant previously to an empty system. Further, we suppose that no customer arrives in the beginning time interval $\left(0^{-}, 0\right)$ and no departure occurs in $\left(0,0^{+}\right)$.

The customer arrival intervals, $\left\{\tau_{i}, i \geq 1\right\}$, are independent identically distributed (i.i.d) random variables following a geometric distribution $P\left\{\tau_{i}=j\right\}=\lambda(1-\lambda)^{j-1}(j \geq 1,0<$ $\lambda<1)$. When the sum of the service times of all waiting customers exceeds a fixed positive integer value $D$, the server restarts service in the first-come, first-served (FCFS) order until the system becomes empty. The service times, denoted by $\left\{\chi_{n}, n \geq 1\right\}$, are i.i.d random variables obeying an arbitrary distribution $g_{j}=P\left\{\chi_{n}=j\right\}, j \geq 1$, with probability generating function (PGF) $G(z)=\sum_{j=1}^{\infty} g_{j} z^{j},|z|<1$ and mean $\mu$. The customer whose service has been just completed leaves forever with probability $\theta(0<\theta \leq 1)$ or returns to the queue with complementary probability $1-\theta$ for the next service. At initial epoch $0^{+}$, the server is idle and then begins to serve the first arrival if the number of customers in the system $N\left(0^{+}\right)=0$ or immediately begins to serve if $N\left(0^{+}\right)>0$. After the first busy period, the server will take a modified D-policy. All random variables involved in the system are independent of one another.

For later discussion, some preliminaries are presented as follows.

2.1. Total Service Time of One Customer before Departure. Let $\chi_{n}^{i}, \widehat{\chi}_{n}$, and $\Omega_{n}$ denote the $i$ th service time, total service time, and total service number of customer $n$ before departure, respectively. Then according to queue assumptions we get $P\left(\Omega_{n}=k\right)=\theta(1-\theta)^{k-1}, k \geq 1$, and $\widehat{\chi}_{n}=\sum_{i=1}^{\Omega_{n}} \chi_{n}^{i}, n \geq 1$ are i.i.d. random variables, where $\chi_{n}^{i}, i, n \geq 1$ are mutually independent and have the same distribution as $\chi_{n}, n \geq 1$.

Further, the probability distribution $\widehat{g}_{j}$, PGF $\widehat{G}(z)$, and mean $E(\widehat{\chi})$ of total service time $\widehat{\chi}$ for one customer before departure are given, respectively, by

$$
\begin{gathered}
\widehat{g}_{j}=P(\widehat{\chi}=j)=P\left\{\sum_{i=1}^{\Omega_{n}} \chi_{n}^{i}=j\right\} \\
=\sum_{k=1}^{j} \theta(1-\theta)^{k-1} P\left\{\sum_{i=1}^{k} \chi_{n}^{i}=j\right\}, \\
\widehat{G}(z)=\sum_{j=1}^{\infty} \widehat{g}_{j} z^{j}=\frac{\theta G(z)}{1-(1-\theta) G(z)}, \quad|z|<1, \\
E(\widehat{\chi})=\left.\frac{\mathrm{d} \widehat{G}(z)}{\mathrm{d} z}\right|_{z=1}=\frac{\mu}{\theta} .
\end{gathered}
$$

2.2. The System Idle Period. It denotes the time length from the time when the system becomes empty until the first customer arrives and enters the system.

Let $I_{i}$ denote the $i$ th system idle period initiated with the initial state $N\left(0^{+}\right)(\geq 0)$; then it follows from the queue assumptions that $\left\{I_{i}, i \geq 1\right\}$ are independent random variables following an identical geometric distribution with parameter $\lambda$.

2.3. The Server Busy Period. It denotes the time length from the time when the server begins to serve until the system becomes empty.

Denote by $b$ the server busy period initiated with one customer and its PGF is given by $b(z)=\sum_{j=0}^{\infty} P(b=j) z^{j}$, 
$|z|<1$; then similar to the analysis in [25], $b(z)$ is the root of the equation $b(z)=\widehat{G}[(1-\lambda) z+\lambda b(z) z]$, and the mean is

$$
E(b)= \begin{cases}\frac{\mu}{(\theta-\lambda \mu)}, & \rho<1, \\ \infty, & \rho \geq 1,\end{cases}
$$

where $\rho=\lambda \mu / \theta$ represents the traffic intensity of the considered queue.

Let $b^{\langle i\rangle}$ denote the server busy period initiated with $i$ customers; then $b^{\langle i\rangle}$ can be expressed as $b^{\langle i\rangle}=\varsigma_{1}+\cdots+$ $\varsigma_{i}$, where $\varsigma_{1}, \ldots, \varsigma_{i}$ are mutually independent with the same distribution as $b$. So the PGF of $b^{\langle i\rangle}$ is given by $b^{i}(z),|z|<1$.

\section{The Analysis of System Size Distributions}

In this section, by a decomposition method, we first analyze the PGF of transient system size distribution. Then based on this PGF we obtain the steady-state system size distribution at epoch $n^{+}$. Lastly we derive the steady-state system size distributions at epochs $n^{-}$and $n$.

3.1. PGF for Transient System Size Distribution at Epoch $n^{+}$in Server Busy Period b. Denote by $Q_{j}\left(n^{+}\right)=P\left\{b>n^{+}, N\left(n^{+}\right)=\right.$ $j$ \}, $j \geq 1$ transient system size distribution at epoch $n^{+}$in server busy period $b$, and its PGF is $q_{j}(z)=\sum_{n=0}^{\infty} Q_{j}\left(n^{+}\right) z^{n}$, $|z|<1$.

Let $\widehat{\chi}_{b}$ be the total service time of the first customer served in server busy period $b$, and $J$ denotes the arrival number during $\widehat{\chi}_{b}$. Since the length of $b$ is irrelevant to the service order of customers and the customer arrival is a Bernoulli process with rate $\lambda$, we get

$$
P(J=j)=\sum_{k=j}^{\infty} \widehat{g}_{k} C_{k}^{j} \lambda^{j}(1-\lambda)^{k-j}, \quad j \geq 0 .
$$

Denote by $\mathrm{O}_{1}, \mathrm{O}_{2}, \ldots, \mathrm{O}_{J}$ the $J$ arrivals during $\widehat{\chi}_{b}$, called primary customers. Those who arrive after the primary customers are called secondary customers. Since the service order of customers has no influence on the length of $b$, we can apply the following service order: primary customers are served in the order of $\mathrm{O}_{1}, \mathrm{O}_{2}, \ldots, \mathrm{O}_{J}$. After serving each primary customer, the server will serve any secondary customer until there are no secondary customers present. Therefore we have the decomposition of $b$ as follows:

$$
b=\widehat{\chi}_{b}+l_{1}+\cdots+l_{J},
$$

where $l_{k}, 1 \leq k \leq J$, denotes the time length from the epoch when the server begins to serve the $k$ th primary customer until the next epoch when the service of the $(k+$ $1)$ th primary customer begins. Thus, $l_{k}(k=1,2, \ldots, J)$ is mutually independent and has the same distribution as $b$. It is clear that $l_{1}+l_{2}+\cdots+l_{J}=0$ if $J=0$. Noting that the point when the server busy period ends is a regenerative one, for $j \geq 1$, we get

$$
\begin{aligned}
Q_{j}\left(n^{+}\right) & \\
= & P\left\{\widehat{\chi}_{b}+l_{1}+\cdots+l_{J}>n^{+}, N\left(n^{+}\right)=j\right\} \\
= & P\left\{\widehat{\chi}_{b}>n^{+}, N\left(n^{+}\right)=j\right\} \\
& +P\left\{\widehat{\chi}_{b} \leq n^{+}<\widehat{\chi}_{b}+l_{1}+\cdots+l_{J}, N\left(n^{+}\right)=j\right\} \\
= & \sum_{k=n+1}^{\infty} \widehat{g}_{k} C_{n}^{j-1} \lambda^{j-1}(1-\lambda)^{n-j+1} \\
& +\sum_{k=1}^{n} \widehat{g}_{k} \sum_{i=0}^{k} C_{k}^{i} \lambda^{i}(1-\lambda)^{k-i} \\
& \quad \times P\left\{l_{1}+\cdots+l_{i}>(n-k)^{+}, N\left((n-k)^{+}\right)=j\right\} .
\end{aligned}
$$

By the above service order, if the epoch $(n-k)^{+}$is located in $l_{m}(1 \leq m \leq i)$ and $N\left((n-k)^{+}\right)=j$, then at epoch $(n-k)^{+}$ there are $i-m$ primary customers and $j-(i-m)$ secondary customers in the system. From the fact that $l_{m}(1 \leq m \leq i)$ obeys the same probability property as $b$ and total probability formula, we obtain

$$
\begin{aligned}
& P\left\{l_{1}+\cdots+l_{i}>(n-k)^{+}, N\left((n-k)^{+}\right)=j\right\} \\
& =\sum_{m=1}^{i} P\left\{l_{1}+\cdots+l_{m-1} \leq(n-k)^{+}\right. \\
& \left.<l_{1}+\cdots+l_{m}, N\left((n-k)^{+}\right)=j\right\} \\
& =\sum_{m=1}^{i} \sum_{r=m-1}^{n-k} P\left\{l_{1}+\cdots+l_{m-1}=r\right\} \\
& \times P\left\{l_{m}>(n-k-r)^{+},\right. \\
& =\sum_{m=1}^{i} \sum_{r=m-1}^{n-k} P\left\{l_{1}+\cdots+l_{m-1}=r\right\} \\
& \times Q_{j-i+m}\left((n-k-r)^{+}\right) .
\end{aligned}
$$

Substituting (6) into (5) and taking the PGF of (5), we get the expression of $q_{j}(z), j=1,2, \ldots$, as follows:

$$
\begin{aligned}
q_{j}(z) & \\
= & \frac{1}{\widehat{G}((1-\lambda) z)} \\
& \times\left\{\sum_{i=1}^{j-1} \frac{q_{j-i}(z)}{b^{i}(z)}\right.
\end{aligned}
$$




$$
\begin{aligned}
& \times[b(z)-\widehat{G}((1-\lambda) z) \\
& \left.-\sum_{m=1}^{i} \sum_{k=m}^{\infty} C_{k}^{m} \widehat{g}_{k} z^{k}(\lambda b(z))^{m}(1-\lambda)^{k-m}\right] \\
& \left.+b(z) \sum_{n=j-1}^{\infty} z^{n} \sum_{k=n+1}^{\infty} \widehat{g}_{k} C_{n}^{j-1} \lambda^{j-1}(1-\lambda)^{n-j+1}\right\},
\end{aligned}
$$

$|z|<1$

where $\sum_{i=1}^{j}=0$ if $j \leq 0$.

\subsection{PGF for Conditional Distribution of Transient System} Size at Any Epoch $n^{+}$. Denote by $P_{i j}\left(n^{+}\right)=P\left\{N\left(n^{+}\right)=\right.$ $\left.j \mid N\left(0^{+}\right)=i\right\}, i, j=0,1,2, \ldots$ the conditional distribution of transient system size at epoch $n^{+}$, and $p_{i j}(z)=$ $\sum_{n=0}^{\infty} P_{i j}\left(n^{+}\right) z^{n},|z|<1$. Also, we denote $\alpha_{m}=\beta_{m-1}-\beta_{m}$, $\beta_{m}=P\left\{\sum_{j=1}^{m} \widehat{\chi}_{j}<D\right\}, m=1,2, \ldots, D, \beta_{0}=1, \beta_{D}=0$.

Let $I_{k}$ and $b_{k}, k \geq 1$ denote the $k$ th system idle period and server busy period initiated with initial state $N\left(0^{+}\right)=0$, respectively; then from the assumption that after the first busy period the server takes a modified D-policy, $b_{1}$ obeys the same distribution as $b$ (see Section 2.3). Noting that $N\left(n^{+}\right)=0$ if and only if the epoch $n^{+}$is located in system idle period, we get

$$
\begin{aligned}
& P_{00}\left(n^{+}\right) \\
& =P\left\{0 \leq n^{+}<I_{1}\right\} \\
& \quad+P\left\{I_{1}+b_{1} \leq n^{+}<I_{1}+b_{1}+I_{2}\right\} \\
& \quad+\sum_{m=1}^{D} P\left\{\sum_{j=1}^{m-1} \widehat{\chi}_{j}<D \leq \sum_{j=1}^{m} \widehat{\chi}_{j},\right. \\
& \left.I_{1}+b_{1}+I_{2}+\sum_{k=1}^{m-1} \tau_{k} \leq n^{+}, N\left(n^{+}\right)=0\right\} .
\end{aligned}
$$

Since the customer arrivals are generated by Bernoulli process, the ending epoch of server busy period $b_{1}$ is a renewal point. So the second term of (8) is equal to

$$
\sum_{u=2}^{n} P\left\{I_{1}+b_{1}=u\right\}(1-\lambda)^{n-u}
$$

The $\sum_{j=1}^{m-1} \widehat{\chi}_{j}<D \leq \sum_{j=1}^{m} \widehat{\chi}_{j}$ in the third term of (8) means that there are $m$ arrivals at initiation point of the second busy period $b_{2}$ of server. As the beginning epoch of $b_{2}$ is a renewal point, using renewal process theory, the third term of (8) is expressed as

$$
\begin{aligned}
\sum_{m=1}^{D} \alpha_{m} & \sum_{u=2}^{n-m} P\left\{I_{1}+b_{1}=u\right\} \\
& \times \sum_{t=1}^{n-u-m+1} P\left\{I_{2}=t\right\} \sum_{r=m-1}^{n-u-t} P\left\{\sum_{k=1}^{m-1} \tau_{k}=r\right\} \\
& \times P_{m 0}\left((n-u-t-r)^{+}\right) .
\end{aligned}
$$

Multiplying (8) by $z^{n}$ and summing over $n$ after substituting (9) and (10) into (8), it finally yields

$$
\begin{aligned}
p_{00}(z)= & \frac{1-f(z)}{1-z}+\frac{f(z) b(z)(1-f(z))}{1-z} \\
& +b(z) \sum_{m=1}^{D} \alpha_{m}[f(z)]^{m+1} p_{m 0}(z),
\end{aligned}
$$

where $f(z)=\lambda z /(1-(1-\lambda) z)$, and $b(z)$ is given by Section 2.3.

For $i \geq 1, P_{i 0}\left(n^{+}\right)$shows that there are $i$ customers at initial epoch $0^{+}$. Based on the supposition in Section 1, the server busy period $b^{\langle i\rangle}$ will begin at epoch $0^{+}$. Similar to the analysis of $P_{00}\left(n^{+}\right)$, so we have

$$
\begin{aligned}
P_{i 0}\left(n^{+}\right) & \\
= & P\left\{b^{\langle i\rangle} \leq n^{+}<b^{\langle i\rangle}+I_{1}\right\} \\
& +\sum_{m=1}^{D} P\left\{\sum_{j=1}^{m-1} \widehat{\chi}_{j}<D \leq \sum_{j=1}^{m} \widehat{\chi}_{j},\right. \\
= & \left.\sum_{u=}^{n} P\left\{b^{\langle i\rangle}=u\right\}(1-\lambda)^{n-u}+I_{1}+\sum_{k=1}^{m-1} \tau_{k} \leq n^{+}, N\left(n^{+}\right)=0\right\} \\
& +\sum_{m=1}^{D} \alpha_{m} \sum_{u=i}^{n-m} P\left\{b^{\langle i\rangle}=u\right\} \\
& \times \sum_{t=1}^{n-u-m+1} P\left\{I_{1}=t\right\} \times \sum_{r=m-1}^{n-u-t} P\left\{\sum_{k=1}^{m-1} \tau_{k}=r\right\} \\
& \times P_{m 0}\left((n-u-t-r)^{+}\right) .
\end{aligned}
$$

Taking the PGF on (12) gives

$$
\begin{aligned}
p_{i 0}(z)= & \frac{b^{i}(z)(1-f(z))}{1-z} \\
& +b^{i}(z) \sum_{m=1}^{D} \alpha_{m} f^{m}(z) p_{m 0}(z),
\end{aligned}
$$


Solving (11) and (13) yields

$$
\begin{aligned}
& p_{00}(z)=\frac{1-f(z)}{1-z}\left\{1+\frac{f(z) b(z)}{1-\sum_{m=1}^{D} \alpha_{m}[f(z) b(z)]^{m}}\right\}, \\
& p_{i 0}(z)=\frac{[1 \mid<1,}{(1-z)\left\{1-\sum_{m=1}^{D} \alpha_{m}[f(z) b(z)]^{m}\right\}},
\end{aligned}
$$$$
|z|<1, i \geq 1 \text {. }
$$

For $1 \leq j \leq D-1, N\left(n^{+}\right)=j$ represents the fact that the epoch $n^{+}$is in server busy period or server idle period with $j$ customers being at present. By means of the same analysis method used for $P_{00}\left(n^{+}\right)$, we have

$$
\begin{aligned}
& P_{0 j}\left(n^{+}\right) \\
& =P\left\{I_{1} \leq n^{+}<I_{1}+b_{1}, N\left(n^{+}\right)=j\right\} \\
& \quad+P\left\{\sum_{i=1}^{j} \widehat{\chi}_{i}<D,\right. \\
& +\sum_{m=1}^{D}\left\{\sum_{i=1}^{m-1} \widehat{\chi}_{i}<D \leq \sum_{i=1}^{m} \widehat{\chi}_{i},\right. \\
& \left.I_{1}+b_{1}+I_{2}+\sum_{l=1}^{j-1} \tau_{l} \leq n^{+}<I_{1}+b_{1}+I_{2}+\sum_{l=1}^{j} \tau_{l}\right\} \\
& \left.\quad \sum_{l=1}^{m-1} \tau_{l} \leq n^{+}, N\left(n^{+}\right)=j\right\} .
\end{aligned}
$$

In the first term of (15), the expression of " $I_{1} \leq n^{+}<I_{1}+$ $b_{1}, N\left(n^{+}\right)=j$ " denotes the epoch $n^{+}$located in the first server busy period $b_{1}$ and the customer number in system is $j$. From the fact that the beginning point of $b_{1}$ is a renewal one, the first term of (15) is

$$
\sum_{u=1}^{n} P\left\{I_{1}=u\right\} Q_{j}\left((n-u)^{+}\right) .
$$

In the second term of (15), the " $\sum_{i=1}^{j} \widehat{\chi}_{i}<D, I_{1}+b_{1}+I_{2}+$ $\sum_{l=1}^{j-1} \tau_{l} \leq n^{+}<I_{1}+b_{1}+I_{2}+\sum_{l=1}^{j} \tau_{l}$ " implies that the first arrival occurs during the second system idle period $I_{2}$, the epoch $n^{+}$ is located in the $j$ th arrival-interval $\tau_{j}, j \geq 1$ after $I_{2}$, and at initiation point of $b_{2}$ total arrival number exceeds $j$. It means that there are total $j$ customers in the system at epoch $n^{+}$but the service does not begin, so the second term of (15) is given by

$$
\beta_{j} \sum_{u=j+2}^{n} P\left\{I_{1}+b_{1}+I_{2}+\sum_{l=1}^{j-1} \tau_{l}=u\right\} P\left\{\tau_{j}>(n-u)^{+}\right\} .
$$

Being similar to the analysis of (10), the third term of (15) is given by

$$
\sum_{m=1}^{D} \alpha_{m} \sum_{u=m+2}^{n} P\left\{I_{1}+b_{1}+I_{2}+\sum_{l=1}^{m-1} \tau_{l}=u\right\} P_{m j}\left((n-u)^{+}\right) .
$$

Multiplying (15) by $z^{n}$ and summing over $n$ after substituting (16)-(18) into (15), we obtain, for $j=1,2, \ldots, D-1$,

$$
\begin{aligned}
p_{0 j}(z)= & f(z) q_{j}(z)+\frac{1-f(z)}{1-z} f^{j+1}(z) b(z) \beta_{j} \\
& +b(z) \sum_{m=1}^{D} \alpha_{m} f^{m+1}(z) p_{m j}(z) .
\end{aligned}
$$

For $i \geq 1$, similarly, we have

$$
\begin{aligned}
& P_{i j}\left(n^{+}\right) \\
& =P\left\{b^{\langle i\rangle}>n^{+}, N\left(n^{+}\right)=j\right\} \\
& +\beta_{j} \sum_{u=j+i}^{n} P\left\{b^{\langle i\rangle}+I_{1}+\sum_{l=1}^{j-1} \tau_{l}=u\right\} \\
& \quad \times P\left\{\tau_{j}>(n-u)^{+}\right\} \\
& +\sum_{m=1}^{D} \alpha_{m} \sum_{u=m+i}^{n} P\left\{b^{\langle i\rangle}+I_{1}+\sum_{l=1}^{m-1} \tau_{l}=u\right\} \\
& \times P_{m j}\left((n-u)^{+}\right) .
\end{aligned}
$$

Since $b^{\langle i\rangle}$ can be expressed by $b^{\langle i\rangle}=\varsigma_{1}+\cdots+\varsigma_{i}$, where $\varsigma_{1}, \ldots, \varsigma_{i}$ are i.i.d. variables with the same distribution as the server busy period $b$, applying the decomposition technique used in (6), the first term in (20) is given by

$$
\sum_{k=1}^{i} \sum_{u=k-1}^{n} P\left\{b^{\langle k-1\rangle}=u\right\} Q_{j-i+k}\left((n-u)^{+}\right)
$$

Substituting (21) into (20), multiplying (20) by $z^{n}$, and summing over $n$ yield

$$
\begin{aligned}
p_{i j}(z)= & \sum_{k=1}^{i} q_{j-i+k}(z) b^{k-1}(z) \\
& +\frac{1-f(z)}{1-z} f^{j}(z) b^{i}(z) \beta_{j} \\
& +b^{i}(z) \sum_{m=1}^{D} \alpha_{m} f^{m}(z) p_{m j}(z) .
\end{aligned}
$$


Solving (19) and (22) leads to the expressions of $p_{0 j}(z)$ and $p_{i j}(z)$; that is, for $|z|<1, i \geq 1$, and $j=1,2, \ldots, D-1$,

$$
\begin{aligned}
& \begin{array}{l}
p_{0 j}(z) \\
=
\end{array} \quad f(z) q_{j}(z) \\
& \quad+\frac{((1-f(z)) /(1-z)) f^{j+1}(z) b(z) \beta_{j}+f(z) \varphi(z)}{1-\sum_{m=1}^{D} \alpha_{m}[f(z) b(z)]^{m}}, \\
& p_{i j}(z) \\
& =\sum_{k=1}^{i} q_{j-i+k}(z) b^{k-1}(z) \\
& \quad+\frac{((1-f(z)) /(1-z)) f^{j}(z) b^{i}(z) \beta_{j}+b^{i-1}(z) \varphi(z)}{1-\sum_{m=1}^{D} \alpha_{m}[f(z) b(z)]^{m}},
\end{aligned}
$$

where $\varphi(z)=\sum_{m=1}^{D} \alpha_{m} f^{m}(z) \sum_{k=1}^{m} b^{k}(z) q_{j-m+k}(z)$.

Finally, since $N\left(n^{+}\right)=j, j \geq D$ indicates that the epoch $n^{+}$ is in server busy period, by similar operations in the analysis of $p_{i j}(z), i \geq 0, j=1,2, \ldots, D-1$, we obtain, for $|z|<1, i \geq 1$, and $j=D, D+1, D+2, \ldots$,

$$
\begin{aligned}
& p_{0 j}(z) \\
& \quad=f(z) q_{j}(z) \\
& \quad+\frac{f(z) \sum_{m=1}^{D} \alpha_{m} f^{m}(z) \sum_{k=1}^{m} b^{k}(z) q_{j-m+k}(z)}{1-\sum_{m=1}^{D} \alpha_{m}[f(z) b(z)]^{m}}, \\
& p_{i j}(z) \\
& \quad \sum_{k=1}^{i} q_{j-i+k}(z) b^{k-1}(z) \\
& \quad+\frac{b^{i-1}(z) \sum_{m=1}^{D} \alpha_{m} f^{m}(z) \sum_{k=1}^{m} b^{k}(z) q_{j-m+k}(z)}{1-\sum_{m=1}^{D} \alpha_{m}[f(z) b(z)]^{m}} .
\end{aligned}
$$

3.3. Steady-State System Size Distribution at Any Epoch $n^{+}$. Now, based on the PGF of the transient-state system size distribution obtained above, we investigate the steady-state system size distribution at any epoch $n^{+}$.

Let $p_{j}^{+}=\lim _{n \rightarrow \infty} P\left\{N\left(n^{+}\right)=j\right\}, j \geq 0$. According to the law of total probability and limitation theory of $z$-transform (see [24]), we get

$$
\begin{aligned}
p_{j}^{+} & =\lim _{n \rightarrow \infty} \sum_{i=0}^{\infty} P_{i j}\left(n^{+}\right) P\left\{N\left(0^{+}\right)=i\right\} \\
& =\sum_{i=0}^{\infty} P\left\{N\left(0^{+}\right)=i\right\} \lim _{n \rightarrow \infty} P_{i j}\left(n^{+}\right) \\
& =\lim _{z \uparrow 1}(1-z) p_{i j}(z) .
\end{aligned}
$$

In (25), applying the expressions of $p_{i j}(z), i, j \geq 0$, L'Hospital's rule, and (2) leads to

(1) for $\rho=\lambda \mu / \theta \geq 1, p_{j}^{+}=0, j=0,1,2, \ldots$;
(2) for $\rho=\lambda \mu / \theta<1$, the steady-state system size distribution $\left\{p_{j}^{+}, j \geq 0\right\}$ is given by

$$
\begin{gathered}
p_{0}^{+}=\frac{1-\rho}{1+\sum_{j=1}^{D-1} \beta_{j}}, \\
p_{j}^{+}=p_{0}^{+}\left(\beta_{j}+\sum_{m=1}^{D} \alpha_{m} \sum_{k=1}^{m} \lambda q_{j-m+k}(1)\right), \\
j=1,2, \ldots, D-1, \\
p_{j}^{+}=p_{0}^{+} \sum_{m=1}^{D} \alpha_{m} \sum_{k=1}^{m} \lambda q_{j-m+k}(1), \\
j=D, D+1, \ldots,
\end{gathered}
$$

where

$$
q_{1}(1)=\frac{1-\widehat{G}(1-\lambda)}{\lambda \widehat{G}(1-\lambda)},
$$

$q_{j}(1)$

$$
\begin{aligned}
& =\frac{1}{\widehat{G}(1-\lambda)} \\
& \quad \times\left\{\sum_{n=j-1}^{\infty} \sum_{k=n+1}^{\infty} \widehat{g}_{k} C_{n}^{j-1} \lambda^{j-1}(1-\lambda)^{n-j+1}\right. \\
& \\
& \quad+\sum_{i=1}^{j-1} q_{j-i}(1)[1-\widehat{G}(1-\lambda) \\
& \left.\left.\quad-\sum_{m=1}^{i} \sum_{k=m}^{\infty} \widehat{g}_{k} C_{k}^{m} \lambda^{m}(1-\lambda)^{k-m}\right]\right\},
\end{aligned}
$$

Remark 1. When $\rho=\lambda \mu / \theta<1$, noting that $\sum_{j=1}^{\infty} \sum_{k=1}^{m} q_{j-m+k}(1)=m \rho /(\lambda(1-\rho))$ and $\sum_{m=1}^{D} m \alpha_{m}=$ $1+\sum_{j=1}^{D-1} \beta_{j}$, we easily get $\sum_{j=0}^{\infty} p_{j}^{+}=1$.

Let $\pi^{+}(z)=\sum_{j=0}^{\infty} p_{j}^{+} z^{j},|z|<1$ be the PGF of steady-state system size at epoch $n^{+}$. For $\rho=\lambda \mu / \theta<1$, calculating directly and noting

$$
\begin{aligned}
& \sum_{j=1}^{\infty} z^{j} \sum_{k=1}^{m} q_{j-m+k}(1) \\
& =\frac{z\left(1-z^{m}\right)[1-\widehat{G}(1-\lambda+\lambda z)]}{\lambda(1-z)[\widehat{G}(1-\lambda+\lambda z)-z]} \\
& (1-z)\left(1+\sum_{j=1}^{D-1} \beta_{j} z^{j}\right)=1-\sum_{m=1}^{D} \alpha_{m} z^{m},
\end{aligned}
$$


we have

$$
\begin{aligned}
\pi^{+}(z)= & \frac{(1-\rho)(1-z) \widehat{G}(1-\lambda+\lambda z)}{\widehat{G}(1-\lambda+\lambda z)-z} \\
& \cdot \frac{1+\sum_{j=1}^{D-1} \beta_{j} z^{j}}{1+\sum_{j=1}^{D-1} \beta_{j}} .
\end{aligned}
$$

Remark 2. It is observed from (29) that in our queue the steady-state system size $\Upsilon$ can be decomposed into the sum of two independent random variables: $\Upsilon=\Upsilon_{0}+\Upsilon_{1} . \Upsilon_{0}$ is the steady-state system size of Geo/G/1 queue with Bernoulli feedback and has the PGF as $((1-\rho)(1-z) \widehat{G}(1-\lambda+\lambda z)) /(\widehat{G}(1-$ $\lambda+\lambda z)-z) . \Upsilon_{1}$ is the number of additional customers due to the modified D-policy and obeys the following probability distribution:

$$
\begin{gathered}
P\left(\Upsilon_{1}=0\right)=\frac{1}{1+\sum_{j=1}^{D-1} \beta_{j}}, \\
P\left(\Upsilon_{1}=j\right)=\frac{\beta_{j}}{1+\sum_{j=1}^{D-1} \beta_{j}}, \\
\quad j=1,2, \ldots, D-1 .
\end{gathered}
$$

Remark 3. It should be noted that the stochastic decomposition property of steady-state system size does not hold for the Geo/G/1 queue under the D-policy (see Lee et al. [16]).

Denote by $E\left[L^{+}\right]$the expected steady-state system size at epoch $n^{+}$; then by $E\left[L^{+}\right]=\left.\left(\mathrm{d} \pi^{+}(z) / \mathrm{d} z\right)\right|_{z=1}$ and direct calculations, for $\rho=\lambda \mu / \theta<1$, we can obtain

$$
E\left[L^{+}\right]=\rho+\frac{\lambda^{2} \widehat{G}^{\prime \prime}(1)}{2(1-\rho)}+\frac{\sum_{j=1}^{D-1} j \beta_{j}}{1+\sum_{j=1}^{D-1} \beta_{j}},
$$

where $j \leq 0, \sum_{i=1}^{j}=0$.

3.4. Steady-State System Size Distributions at Epochs $n$ and $n^{-}$. Let $P_{i j}(n)=P\{N(n)=j \mid N(0)=i\}$ and $P_{i j}\left(n^{-}\right)=$ $P\left\{N\left(n^{-}\right)=j \mid N\left(0^{-}\right)=i\right\}, i, j=0,1,2, \ldots$ be the conditional distributions of transient system size at epochs $n$ and $n^{-}$, $p_{j}=\lim _{n \rightarrow \infty} P\{N(n)=j\}$ and $p_{j}^{-}=\lim _{n \rightarrow \infty} P\left\{N\left(n^{-}\right)=j\right\}$, $j=0,1,2, \ldots$ denote steady- state system size distributions at epochs $n$ and $n^{-}, \pi(z)=\sum_{j=0}^{\infty} p_{j} z^{j}, \pi^{-}(z)=\sum_{j=0}^{\infty} p_{j}^{-} z^{j}$, $|z|<1$, and $E(L)$ is the expected steady-state system size at any epoch $n$. Also, it is assumed that no arrival occurs in the beginning time interval $\left(0^{-}, 0\right)$ and no departure takes place in $\left(0,0^{+}\right)$.

From the assumption that no arrival occurs in $\left(0^{-}, 0\right)$ and no departure takes place in $\left(0,0^{+}\right)$, for $i, j \geq 0, n \geq 1$, we get $P\left\{N\left(0^{-}\right)=j\right\}=P\{N(0)=j\}=P\left\{N\left(0^{+}\right)=j\right\}$. So

$$
\begin{aligned}
P_{i j}\left(n^{-}\right) & =P\left\{N\left(n^{-}\right)=j \mid N\left(0^{-}\right)=i\right\} \\
& =P\left\{N\left((n-1)^{+}\right)=j \mid N\left(0^{+}\right)=i\right\}=P_{i j}\left((n-1)^{+}\right) .
\end{aligned}
$$

Taking limit as $n \rightarrow \infty$ under the stability condition, $\rho=$ $\lambda \mu / \theta<1$, we get

$$
\begin{aligned}
& p_{j}^{-}=p_{j}^{+}, \quad j \geq 0, \text { which is equivalent to } \\
& \pi^{-}(z)=\pi^{+}(z), \quad|z|<1 .
\end{aligned}
$$

As the customers arrive in the system according to Bernoulli process, and the departure occurs only in interval $\left(n, n^{+}\right)$, for $\rho=\lambda \mu / \theta<1$, we obtain $p_{0}=(1-\lambda) p_{0}^{-}$, $p_{j}=(1-\lambda) p_{j}^{-}+\lambda p_{j-1}^{-}, j=1,2, \ldots$ By using $(33), \pi^{+}(z)$ in (29), and $E\left(L^{+}\right)$in (31), when $\rho=\lambda \mu / \theta<1$, we have

$$
\begin{gathered}
p_{0}=(1-\lambda) p_{0}^{+}, \quad p_{j}=(1-\lambda) p_{j}^{+}+\lambda p_{j-1}^{+}, \\
j=1,2, \ldots, \\
\pi(z)=\frac{(1-\lambda+\lambda z)(1-\rho)(1-z) \widehat{G}(1-\lambda+\lambda z)}{\widehat{G}(1-\lambda+\lambda z)-z} \\
\cdot \frac{1+\sum_{j=1}^{D-1} \beta_{j} z^{j}}{1+\sum_{j=1}^{D-1} \beta_{j}}, \\
E(L)=\lambda+\rho+\frac{\lambda^{2} \widehat{G}^{\prime \prime}(1)}{2(1-\rho)}+\frac{\sum_{j=1}^{D-1} j \beta_{j}}{1+\sum_{j=1}^{D-1} \beta_{j}} .
\end{gathered}
$$

Remark 4. At epochs $n^{-}, n^{+}$, and $n$, the following relations are clear:

(1) $p_{j}^{-}=p_{j}^{+} \neq p_{j}, j=0,1,2, \ldots$, or $\pi^{-}(z)=\pi^{+}(z) \neq \pi(z)$;

(2) $p_{0}^{-}=p_{0}^{+}>p_{0}, E\left(L^{-}\right)=E\left(L^{+}\right)<E(L)$.

\section{Special Cases}

Case 1. Let $\theta=1$ and $D=1$; then our model becomes a discrete-time Geo/G/1 queue with LAS-DA. In this case, for $\rho=\lambda \mu<1$, we have

$$
\begin{gathered}
p_{0}^{+}=1-\rho, \quad p_{j}^{+}=\lambda(1-\rho) q_{j}(1), \quad j=1,2, \ldots, \\
\pi^{+}(z)=\frac{(1-\rho)(1-z) G(1-\lambda+\lambda z)}{G(1-\lambda+\lambda z)-z}, \\
E\left[L^{+}\right]=\rho+\frac{\lambda^{2}}{2(1-\rho)} G^{\prime \prime}(1),
\end{gathered}
$$

where $q_{j}(1), j=1,2, \ldots$, are determined by (7), in which $\widehat{g}_{j}=g_{j}$ and $\widehat{G}(z)=G(z)$.

Case 2. Let $\theta=1, D=1$, and the service times $\chi_{i}, i \geq 1$ are independent and follow an identical geometric distribution with parameter $\delta(>0)$. In the present queue with early arrival system (EAS) policy, the steady-state system size distribution at any epoch $n$ is given by

$$
p_{j}^{(e)}=\frac{1-\rho}{1-\lambda}\left(\frac{\rho-\lambda}{1-\lambda}\right)^{j}, \quad j=0,1, \ldots, \text { if } \rho=\frac{\lambda}{\delta}<1,
$$

which agrees with that in [24]. 
TABLE 1: Steady-state radio signal number distributions at epochs $n$ and $n^{+}$for a wireless local area network $(\lambda=0.25, \mu=1, \theta=0.4$, and $D=20)$.

\begin{tabular}{|c|c|c|c|c|c|c|c|}
\hline$j$ & $p_{j}$ & $p_{j}^{+}$ & $P\left\{L^{+}>j\right\}$ & $j$ & $p_{j}$ & $p_{j}^{+}$ & $P\left\{L^{+}>j\right\}$ \\
\hline 0 & 0.03270 & 0.04360 & 1.00000 & 13 & 0.00907 & 0.00763 & 0.09753 \\
\hline 1 & 0.07085 & 0.07993 & 0.95639 & 14 & 0.00532 & 0.00455 & 0.08989 \\
\hline 2 & 0.09327 & 0.09772 & 0.87645 & 15 & 0.00340 & 0.00301 & 0.08534 \\
\hline 3 & 0.10115 & 0.10229 & 0.77873 & 16 & 0.00240 & 0.00220 & 0.08232 \\
\hline 4 & 0.10264 & 0.10276 & 0.67643 & 17 & 0.00182 & 0.00169 & 0.08012 \\
\hline 5 & 0.10115 & 0.10061 & 0.57367 & 18 & 0.00139 & 0.00129 & 0.07842 \\
\hline 6 & 0.09636 & 0.09495 & 0.47305 & 19 & 0.00104 & 0.00096 & 0.07713 \\
\hline 7 & 0.08727 & 0.08471 & 0.37810 & 20 & 0.00075 & 0.00068 & 0.07616 \\
\hline 8 & 0.07375 & 0.07009 & 0.29338 & 21 & 0.00051 & 0.00046 & 0.07548 \\
\hline 9 & 0.05728 & 0.05301 & 0.22329 & 22 & 0.00033 & 0.00029 & 0.07502 \\
\hline 10 & 0.04058 & 0.03643 & 0.17027 & 23 & 0.00021 & 0.00018 & 0.07472 \\
\hline 11 & 0.02628 & 0.02290 & 0.133836 & 24 & 0.00013 & 0.00011 & 0.07453 \\
\hline 12 & 0.01577 & 0.01339 & 0.11093 & 25 & 0.00008 & 0.00006 & 0.07442 \\
\hline
\end{tabular}

In fact, denote by $N^{(e)}(n)$ and $N^{(e)}\left(n^{+}\right)$the system sizes at epochs $n$ and $n^{+}$in the present queue with early arrival system (EAS) policy, and $\left\{p_{j}^{(e)}\right\}$ and $\left\{p_{j}^{(e)+}\right\}$ are the steadystate system size distributions at any epochs $n$ and $n^{+}$. By the relation of customer number between late arrival system with delay access (LAS-DA) and early arrival system (EAS), we get $N\left(n^{+}\right)=N^{(e)}\left(n^{+}\right)$. So $p_{j}^{+}=p_{j}^{(e)+}, j \geq 0$. Since the customer arrival is a Bernoulli process with rate $\lambda$, we have

$$
\begin{gathered}
p_{0}^{+}=p_{0}^{(e)+}=(1-\lambda) p_{0}^{(e)} \\
p_{j}^{+}=p_{j}^{(e)+}=(1-\lambda) p_{j}^{(e)}+\lambda p_{j-1}^{(e)}, \quad j \geq 1 .
\end{gathered}
$$

It follows from (41) that, for $\rho=\lambda / \delta<1$ and $|z|<1$, the relation between $\pi^{+}(z)=\sum_{j=0}^{\infty} p_{j}^{+} z^{j}$ and $\pi^{(e)}(z)=\sum_{j=0}^{\infty} p_{j}^{(e)} z^{j}$ is

$$
\pi^{+}(z)=(1-\lambda+\lambda z) \pi^{(e)}(z)
$$

Using $\pi^{+}(z)$ in (38) and noting that the service times obey geometric distribution with mean $1 / \delta$, we obtain

$$
\pi^{+}(z)=\frac{(1-\rho)(1-\lambda+\lambda z)}{1-\lambda+\lambda z-\rho z} .
$$

From (42) and (43), we get

$$
\begin{aligned}
\sum_{j=0}^{\infty} p_{j}^{(e)} z^{j} & =\pi^{(e)}(z) \\
& =\frac{1-\rho}{1-\lambda+\lambda z-\rho z}=\frac{1-\rho}{1-\lambda} \sum_{j=0}^{\infty}\left(\frac{\rho-\lambda}{1-\lambda}\right)^{j} z^{j} .
\end{aligned}
$$

This gives rise to (40).

\section{Numerical Examples}

One example of an application fitting our model is the wireless local area network (WLAN) introduced in Section 1. In such a system, because the receptions and transmissions of radio signals are proceeded in fixed time intervals, the arrival of radio signals can be modelled as a Bernoulli process with rate $\lambda$, and the transmission time of each radio signal follows a discrete-time distribution (for numerical analysis, we assume that the transmission time is geometrically distributed with mean $\mu$ ). Because of power saving, the AP is designed to start serving exhaustively whenever the workload of radio signals reaches a predetermined positive integer $D$. If a radio signal is transmitted with errors at the destination, it is retransmitted (feedback) with probability $1-\theta$.

To illustrate the applicability and important application in system capacity design of steady-state system size distributions derived in Section 3, based on the results obtained in (26) and (34), we numerically get the steady-state radio signal number distributions $\left\{p_{j}, j \geq 0\right\}$ and $\left\{p_{j}^{+}, j \geq 0\right\}$ for the set of parameters $(\lambda, \mu, \theta, D)=(0.25,1,0.4,20)$ (see Figure 1$)$. Also, by using (31) and (36), the mean radio signal numbers $E\left(L^{+}\right)$and $E(L)$ and the intensity $\rho$ for the above WLAN are obtained (see Table 1).

Remark 5. With the software of Matlab 7.0, the data in Table 1 are accurate to five decimal places.

From the two curves of Figure 1, we conclude that the steady-state distribution probabilities $\left\{p_{j}, j \geq 0\right\}$ and $\left\{p_{j}^{+}, j \geq 0\right\}$ increase rapidly at an early stage and then decrease as radio signal number becomes large. Moreover, the decrease becomes stable when radio signal number is greater than 15. It is clear that the values of $p_{j}$ and $p_{j}^{+}$have a small difference. Since $p_{j}^{+}$or $p_{j}, j>20$ tends to zero, it is improper for this WLAN to design an enough large space capacity. 


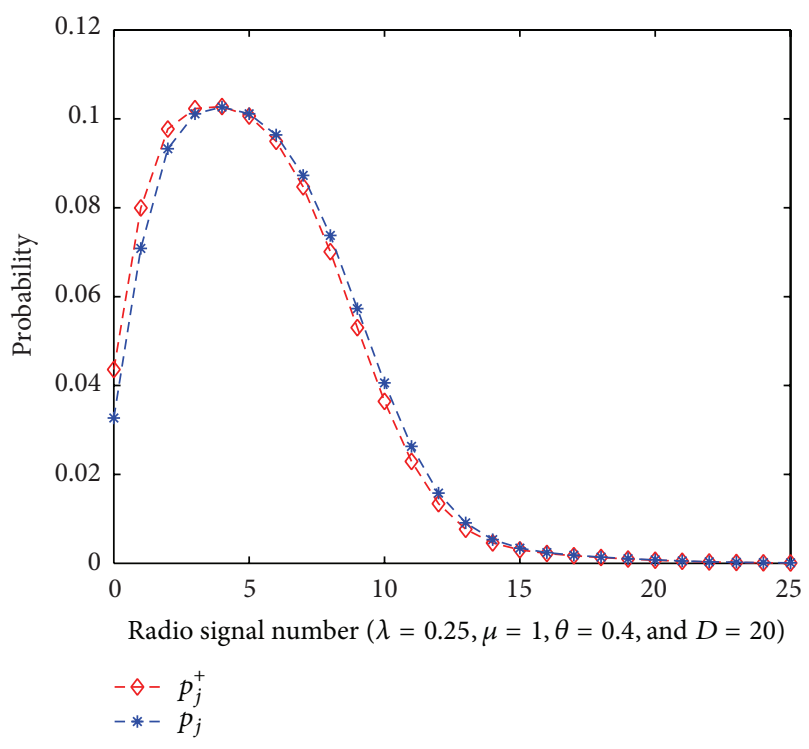

FIGURE 1: Steady-state radio signal number distributions at epochs $n$ and $n^{+}$for a wireless local area network.

Let $K$ denote network space capacity, and $L^{+}$is radio signal number of WLAN at epoch $n^{+}$; then from Table 1 we see that the overflow probability of WLAN $P\left(L^{+}>K\right)$ is smaller for larger network space capacity $K$, which is as expected. Also, from the data in Table 1, we get $P\left\{L^{+}>\right.$ $\left.E\left(L^{+}\right)\right\}=0.57367$. Therefore, it is inappropriate to determine network space capacity with $E\left(L^{+}\right)$because the overflow probability of WLAN is very large.

\section{Conclusions}

This paper studies the steady-state system size distribution for a discrete-time modified D-policy Geo/G/1 queue with feedback. The steady-state system size distributions at threetime epochs are obtained. It should be noted that the steadystate system size distributions presented by this paper are some recursive formulae, which are applicable to accurate numerical calculations. In the future, the system size distribution study of more complex discrete-time queues, such as the modified D-policy Geo ${ }^{X} / G / 1$ queue with feedback and vacation, will be investigated by the similar analysis techniques.

\section{Conflict of Interests}

The authors declare that there is no conflict of interests regarding the publication of this paper.

\section{Acknowledgments}

The authors would like to thank the referees and editor for their valuable comments and suggestions. This work is supported by the Basic and Frontier Research Foundation of Chongqing of China (cstc2013jcyjA00008) and the Scientific Research Starting Foundation for Doctors of Chongqing University of Technology (2012ZD48).

\section{References}

[1] M. Yadin and P. Naor, "Queueing systems with a removable service station," Operational Research Quarterly, vol. 14, no. 3, pp. 393-405, 1963.

[2] D. P. Heyman, “The T-policy for the M/G/1 queue," Management Science, vol. 23, no. 7, pp. 775-778, 1977.

[3] K. R. Balachandran, "Control policies for a single server system," Management Science, vol. 19, no. 9, pp. 1013-1018, 1973.

[4] K. R. Balachandran and H. Tijms, "On the D-policy for the M/G/1 queue," Management Science, vol. 21, no. 9, pp. 1073-1076, 1974/75.

[5] O. J. Boxma, "Note on a control problem of Balachandran and Tijms," Management Science, vol. 22, no. 8, pp. 916-917, 1975/76.

[6] H. C. Tijms, "Optimal control of the workload in an $M / G / 1$ queueing system with removable server," vol. 7, no. 6, pp. 933943, 1976.

[7] J. R. Artalejo, "On the M/G/1 queue with D-policy," Applied Mathematical Modelling, vol. 25, no. 12, pp. 1055-1069, 2001.

[8] K. G. Gakis, H. K. Rhee, and B. D. Sivazlian, "Distributions and first moments of the busy and idle periods in controllable M/G/1 queueing models with simple and dyadic policies," Stochastic Analysis and Applications, vol. 13, no. 1, pp. 47-81, 1995.

[9] H. K. Rhee, "Development of a newmethodology to find the expected busy periods for control- lable M/G/1 queueing models operating under the multivariable operating policies: concepts and applications to the dyadic policies," Journal of Korean Institute of Industrial Engineers, vol. 23, no. 4, pp. 729739,1997

[10] J. Li and S. C. Niu, "The waiting-time distribution for the GI/G/1 queue under the D-policy," Probability in the Engineering and Informational Sciences, vol. 6, no. 3, pp. 287-308, 1992.

[11] R. E. Lillo and M. Martín, "On optimal exhaustive policies for the M/G/1-queue," Operations Research Letters, vol. 27, no. 1, pp. 39-46, 2000.

[12] H. W. Lee and K. S. Song, "Queue length analysis of MAP/G/1 queue under D-policy," Stochastic Models, vol. 20, no. 3, pp. 363380, 2004.

[13] H. W. Lee, J. W. Baek, and J. Jeon, "Analysis of the $M^{X} / G / 1$ queue under D-policy," Stochastic Analysis and Applications, vol. 23, no. 4, pp. 785-808, 2005.

[14] J. H. Dshalalow, "Queueing processes in bulk systems under the D-policy," Journal of Applied Probability, vol. 35, no. 4, pp. 976989, 1998.

[15] R. P. Agarwal and J. H. Dshalalow, "New fluctuation analysis of D-policy bulk queues with multiple vacations," Mathematical and Computer Modelling, vol. 41, no. 2-3, pp. 253-269, 2005.

[16] S. W. Lee, H. W. Lee, and J. W. Baek, "Analysis of discretetime Geo/G/1 queue under the D-policy," in Proceedings of the 6th International Conference on Queueing Theory and Network Applications (QTNA '11), pp. 107-115, August 2011.

[17] S. W. Lee, H. W. Lee, and J. W. Baek, "Analysis of discretetime MAP/G/1 queue under workload control," Performance Evaluation, vol. 69, no. 2, pp. 71-85, 2012.

[18] Y. Wei, Y. Tang, and J. Gu, "Queue size distribution and capacity optimum design for Geo/G/1 queueing system with delayed Dpolicy," System Engineering Theory and Practice, vol. 33, no. 4, pp. 996-1005, 2013.

[19] L. Takács, "A single-server queue with feedback," The Bell System Technical Journal, vol. 42, pp. 505-519, 1963. 
[20] B. D. Choi, B. Kim, and S. H. Choi, "On the M/G/1 Bernoulli feedback queue with multi-class customers," Computers \& Operations Research, vol. 27, no. 3, pp. 269-286, 2000.

[21] D. I. Choi and T.-S. Kim, "Analysis of a two-phase queueing system with vacations and Bernoulli feedback," Stochastic Analysis and Applications, vol. 21, no. 5, pp. 1009-1019, 2003.

[22] B. Krishna Kumar and J. Raja, "On multiserver feedback retrial queues with balking and control retrial rate," Annals of Operations Research, vol. 141, pp. 211-232, 2006.

[23] I. Atencia and P. Moreno, "Discrete-time $G e o^{[X]} / G_{H} / 1$ retrial queue with Bernoulli feedback," Computers \& Mathematics with Applications, vol. 47, no. 8-9, pp. 1273-1294, 2004.

[24] J. J. Hunter, Mathematical Techniques of Applied Probability (II), Academic Press, New York, NY, USA, 1983.

[25] S. W. Fuhrmann and R. B. Cooper, "Stochastic decompositions in the M/G/1 queue with generalized vacations," Operations Research, vol. 33, no. 5, pp. 1117-1129, 1985. 


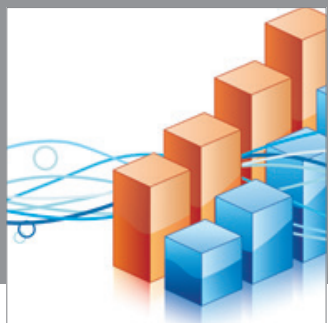

Advances in

Operations Research

mansans

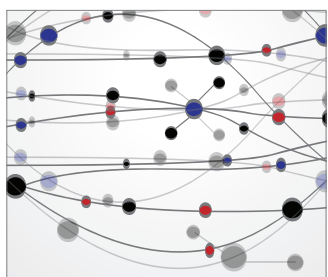

The Scientific World Journal
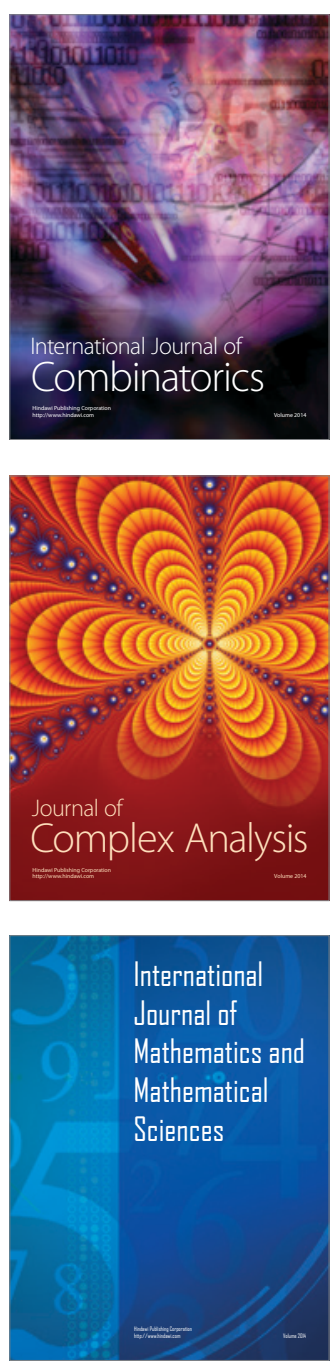
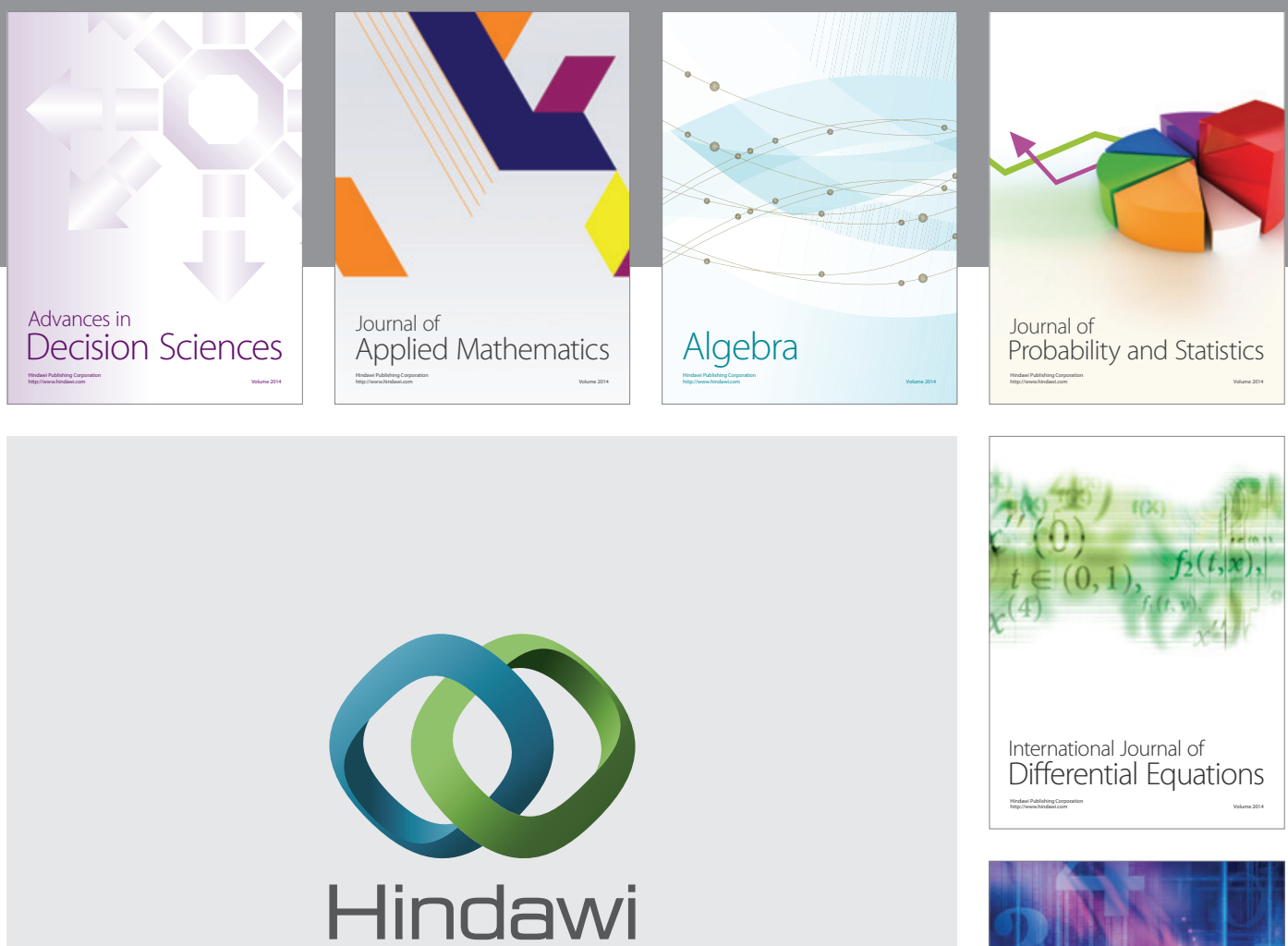

Submit your manuscripts at http://www.hindawi.com
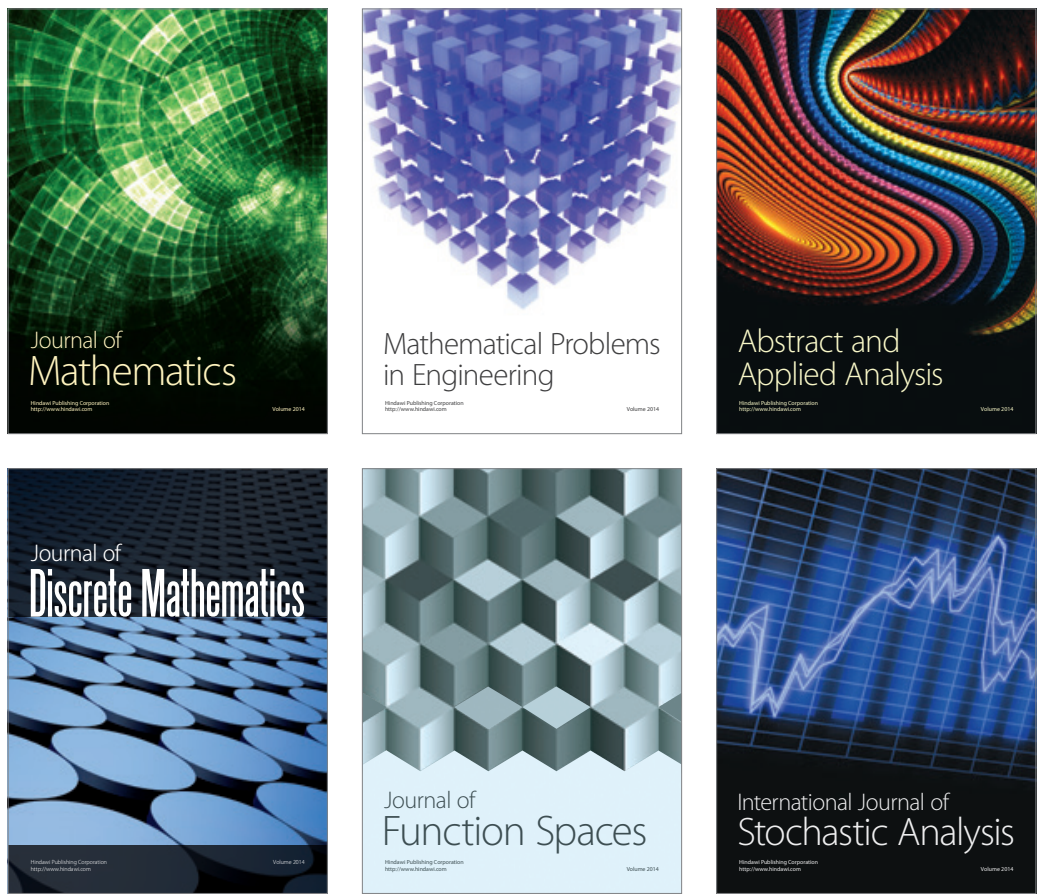

Journal of

Function Spaces

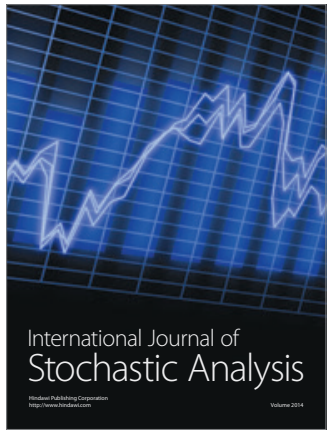

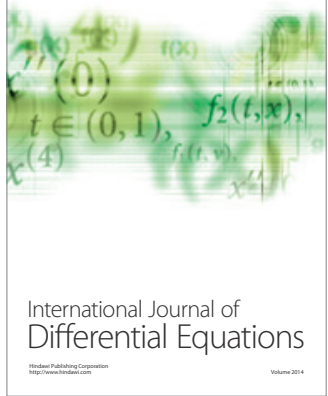
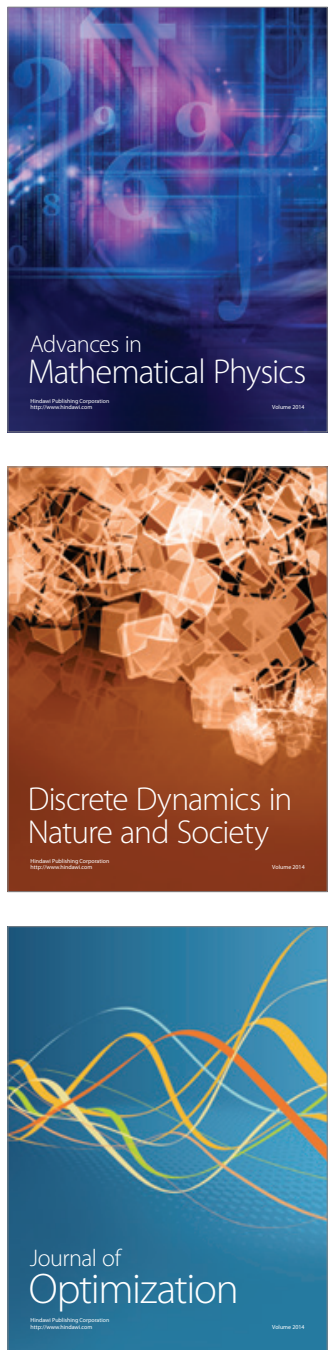